\title{
The Korean Spinal Neurosurgery Society ; Are We Reimbursed Properly for Spinal Neurosurgical Practices under the Korean Resource Based Relative Value Scale Service?
}

\author{
Woo-Keun Kwon, M.D., Joo Han Kim, M.D., Ph.D., Hong Joo Moon, M.D., Ph.D., Youn-Kwan Park, M.D., Ph.D. \\ Department of Neurosurgery, Korea University Guro Hospital, Korea University College of Medicine, Seoul, Korea
}

Objectives : The Korean Resource Based Relative Value Scale (K-RBRVS) was introduced in 2001 as an alternative of the previous medical fee schedule. Unfortunately, most neurosurgeons are unfamiliar with the details of the K-RBRVS and how it affects the reimbursement rates for the surgical procedures we perform. We summarize the K-RBRVS in brief, and discuss on how the relative value (RV) of the spinal neurosurgical procedures have changed since the introduction in 2001.

Methods : We analyzed the change of spinal procedure RVs since 2001, and compared it with the change of values in the brain neurosurgical procedures. RVs of 88 neurospinal procedures on the list of K-RBRVS were analyzed, while 24 procedures added during annual revisions were excluded.

Results : During the past 15 years, RVs for spinal procedures have increased $62.8 \%$, which is not so different with the cumulative increase of consumer prices during this time period or the increase rate of $92.3 \%$ for brain surgeries. When comparing the change of RVs in more complex procedures between spinal and brain neurosurgery, the increase rate was $125.3 \%$ and $133 \%$, respectively.

Conclusion : More effort of the society of spinal surgeons seems to be needed to get adequate reimbursement, as there have been some discrimination compared to brain surgeons in the increase of RVs. And considering the relative underestimation of spinal neurosurgeons' labor, more objective measures of neurospinal surgeons' work and productivity should be developed for impartial reimbursement.

Key Words : Korean Resource Based Relative Value Scale · Relative value · Medical fee schedule · Reimbursement · Neurosurgery.

\section{INTRODUCTION}

Since it's introduction in 1977 , the national health insurance program in Korea has paid health care providers on a fee-for-service basis, and the reimbursement for health care providers has been regulated by the government. Regulated fee-for-service payment has resulted in an increased volume and intensity of medical care ${ }^{5}$. This led to the creation of the

- Received: May 9, 2016 • Accepted: August 29, 2016

- Address for reprints : Youn-Kwan Park, M.D., Ph.D.

Department of Neurosurgery, Korea University Guro Hospital, 148 Gurodongro, Guro-gu, Seoul 08308, Korea

Tel : +82-2-2626-3095, Fax : +82-2-863-1684, E-mail : ykapa76@yahoo.co.kr

This is an Open Access article distributed under the terms of the Creative Commons Attribution Non-Commercial License (http://creativecommons.org/licenses/by-nc/4.0) which permits unrestricted non-commercial use, distribution, and reproduction in any medium, provided the original work is properly cited. 
Korean Resource Based Relative Value Scale (K-RBRVS), which remains in force, with continual evolution, to the current day. K-RBRVS was developed in 1997 based on the RBRVS of the United States of America (USA), which was established in 1992, and was introduced in 2001 as an alternative of the previous Korean medical fee schedule.

Not unexpectedly, most neurosurgeons are unfamiliar with the details of the K-RBRVS and how changes in the KRBRVS affects the reimbursement rates for the surgical procedures we perform. Unfortunately, most physicians might also be unaware of the update process and of the trend of change in relative values (RVs). Physicians in a wide variety of settings may benefit from understanding the K-RBRVS, and from precisely knowing the trend of change in RVs in our surgical specialty. However, to our knowledge no article about this issue in our field has been published yet.

In this article, we summarize the K-RBRVS in brief, and discuss on how the relative value (RV) of the spinal neurosurgical procedures have changed since the introduction of this system in 2001. We also compare these changes with the brain neurosurgical practices, and finally discuss on the fairness regarding whether if we are properly paid or not, for the practices we do in the spinal neurosurgical field.

\section{MATERIALS AND METHODS}

\section{Brief of the K-RBRVS}

During the 1990's, policy makers were concerned about the persistent growth of medical expenditures and at the same time they needed a standardized payment system regarding reimbursement for physicians. They also had to redistribute the income among physicians with different specialties. As a solution, the government decided to introduce the new system from the USA, which was at first developed in order to correct distortions in reimbursement rates between medical practices - some were over-valued while others were relatively under-valued ${ }^{4,6)}$. Since the decision was made in the late 1990's, to import the system from the USA, it took several years to determine the RVs for all physician services in Korea before K-RBRVS was finally implemented in 2001. The KRBRVS, just like the RBRVS of the USA, determines relative fees of physicians' practices on the basis of resource costs required to produce services: total work (time and intensity) of the physician, practice (overhead) costs and the opportunity costs regarding possible risks of professional negligence during medical practices ${ }^{4,5)}$ (Fig. 1). Finally, the payment for a physician's practice is determined by multiplying the RV of each practice by a conversion factor, which converts the RV into Korean Won amounts (Fig. 2).

Since the introduction of K-RBRVS in 2001, it has under-

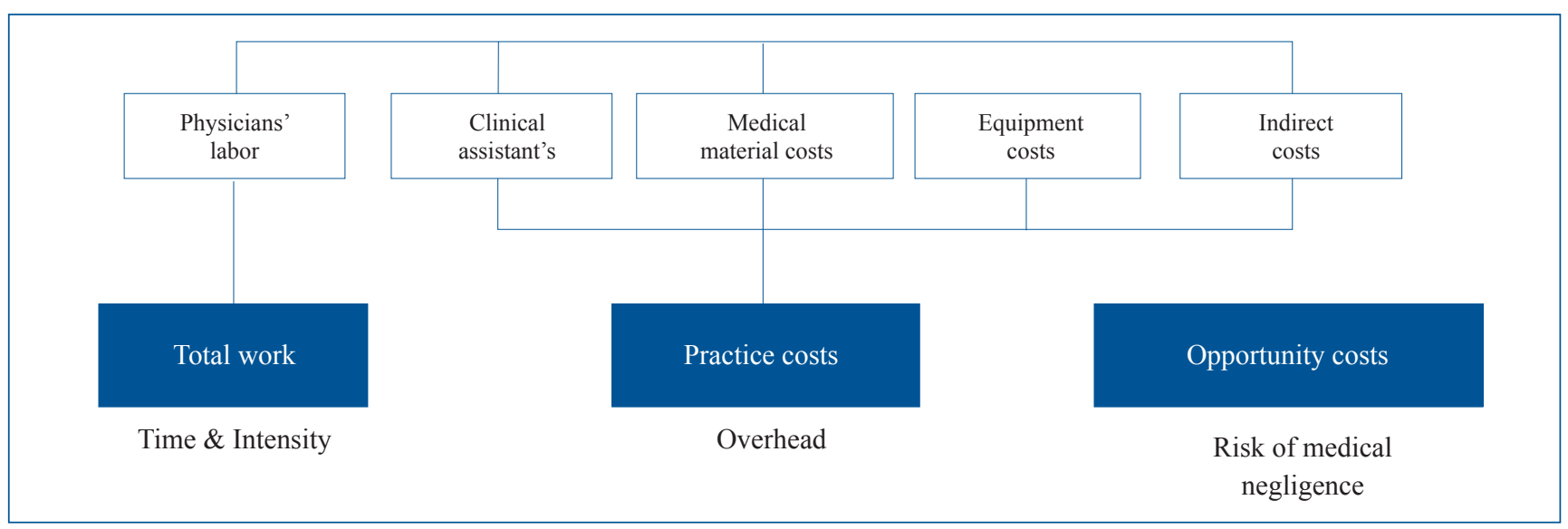

Fig. 1. A schematic box of factors consisting the RV of physician's practice. RVs of physicians' practices are determined on the basis of resource costs required to produce services: total work (time and intensity) of the physician, practice (overhead) costs and the opportunity costs regarding possible risks of professional negligence during medical practices. RV : relative value. 


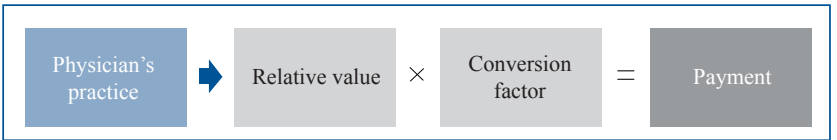

Fig. 2. A schematic box of payment for a physician's practice. Payment for a physician's practice is determined by multiplying the RVs of each practice by a conversion factor, which converts the RV into Korean Won amounts. RV : relative value.

gone several minor revisions and one major revision in 2006 as a reflex of physicians' or the government's requirements, and in order to reflex the actual change in costs as time flows. The system had a major revision on 2006 because, although the objective of the K-RBRVS was to correct the distortion in the structure of the medical fee system, there were still argument on fairness between medical practices of different specialties. This revised version of K-RBRVS was started in 2008 and remains in force until now. Currently, the second major revision of K-RBRVS has been conducted, and is about to be implemented within this year.

\section{RV of spinal neurosurgical practices under K-RBRVS}

We have analyzed the change of spinal neurosurgical procedure RVs since it's first introduction in 2001, and compared it with the change of values in the brain neurosurgical procedures. Twenty-four procedures which were not on the list at 2001, and those that have been added during annual revisions, were excluded from the analysis. Overall average increase in RVs of each neurosurgical fields as well as that of increase in values of complex surgical procedures of both spinal and brain fields are shown on Table 1. While the overall increase rate of RVs of neurospinal surgeries was $62.8 \%$ since 2001 , that of brain surgeries was $92.3 \%$, approximately 30\% points larger. We also compared the RVs of complex surgical procedures in both neurosurgical fields. Complex procedures in each groups were as following ; brain tumor surgeries, arteriovenous malformation surgeries, cerebrovascular aneurysm clipping or vascular bypass surgeries in brain neurosurgery, and vertebral/spinal cord neoplasm surgeries, corpectomy procedures or fusion operations in spinal neurosurgery. When comparing the change of RVs in these complex procedures between spinal neurosurgery and brain neurosurgery, the in-
Table 1. Increase rate of relative values for neurosurgical procedures, during 15 years under the K-RBRVS

\begin{tabular}{lcc}
\hline & Spinal neurosurgery & Brain neurosurgery \\
\hline Overall & $62.8 \%$ & $92.3 \%$ \\
Complex procedures & $125.3 \%$ & $133 \%$ \\
\hline
\end{tabular}

K-RBRVS : Korean Resource Based Relative Value Scale

crease rate was $125.3 \%$ and $133 \%$, respectively. The gap between each fields were smaller than that of the overall increase rate of RVs, however the increase rate of neurospinal practices was still smaller than that of brain surgeries.

Although the increase in RV reimbursement seems quite significant $(62.8 \%)$ in percentages during the past 15 years, when compared to the cumulative increase of consumer price announced by the Korean National Statistical Office during the same period (61.9\%) it is hard to say that the increase is actually significant. And as already mentioned in the prior paragraph, when comparing the increase rate of RV with that of the brain neurosurgical procedures, the increase rate of spinal surgery RVs is even lower. On Fig. 3, the changes in RVs of some representative, index surgery procedures in both neurosurgical fields since 2001, are shown as graph. When comparing complex surgical procedures such as tumor removal, we could find that the RVs have increased consistently since 2001, in both brain and spinal neurosurgery. However, as the RVs of brain tumor surgeries were set at a higher amount of RV at first, the gap of RVs between brain tumor surgeries and spinal cord tumors are still present, resulting in a significantly higher RV for brain tumor surgeries (Fig. 3A). During the past 15 years, RVs of other brain surgical procedures such as vascular anastomosis procedures or aneurysm clipping surgeries have also increased in a high rate, more than $100 \%$. Considering more simple but frequently performed procedures, such as diagnostic or therapeutic craniotomies, the increase rate was $60-100 \%$ which was relatively lower than complex surgeries (Fig. 3B). However, this rate was still a relatively high increase when compared with the frequent procedures performed in spinal neurosurgical field. Complex decompression procedures such as vertebral corpectomy or fusion techniques such as anterior cervical fixation which has relatively high risk of complications 

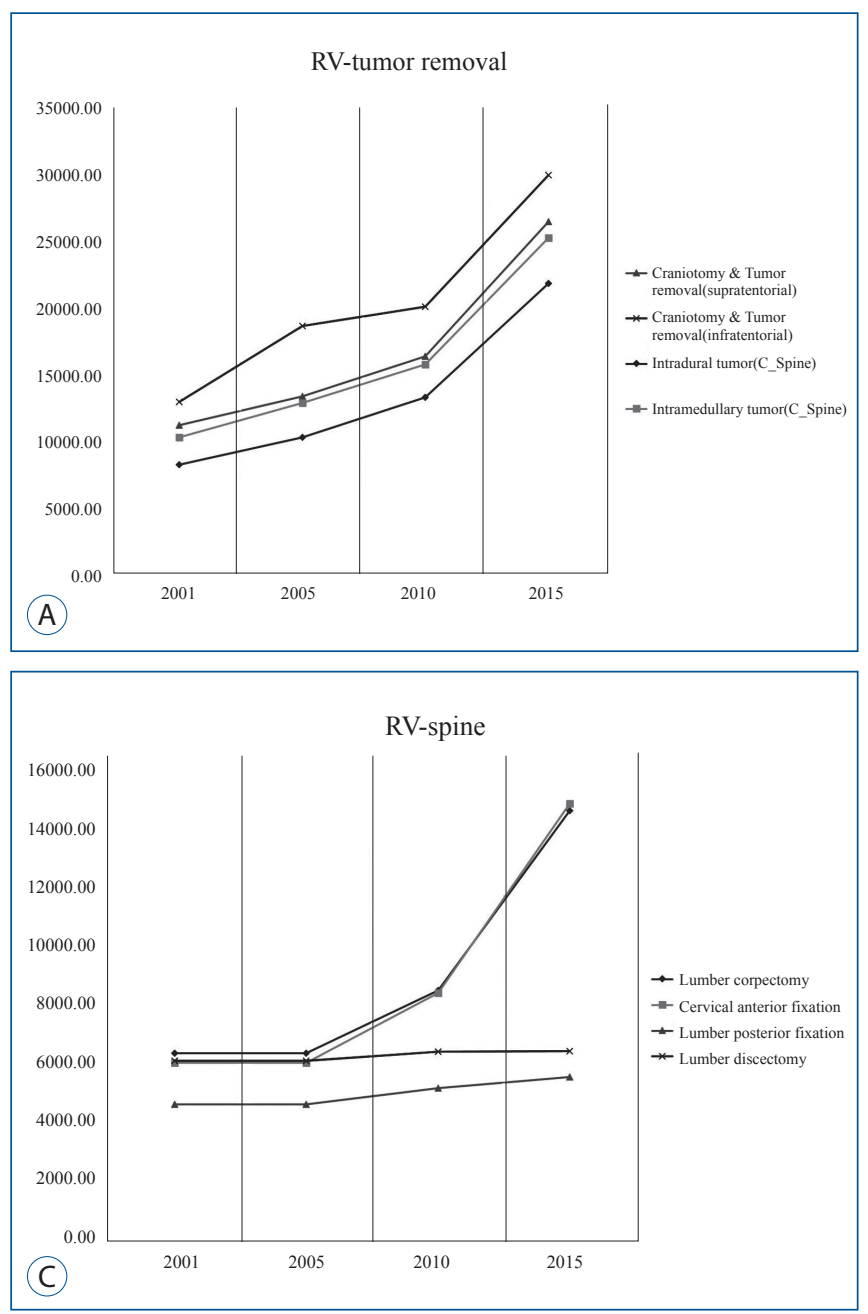

showed increase rate of RVs similar to that of brain procedures. But at the same period of time, more commonly performed surgical procedures such as lumbar posterior fusion or lumbar laminectomy and discectomy showed almost no increase in RV for 15 years (Fig. 3C). Considering the fact that the cumulative increase of consumer price during this period of time was over $60 \%$, these results could mean that the actual RVs of these lumbar procedures have actually decreased.

\section{DISCUSSION}

In this study, in order to estimate whether we are reimbursed for surgical practices properly or not by the K-RBRVS, we have analyzed the RV of spinal neurosurgical practices

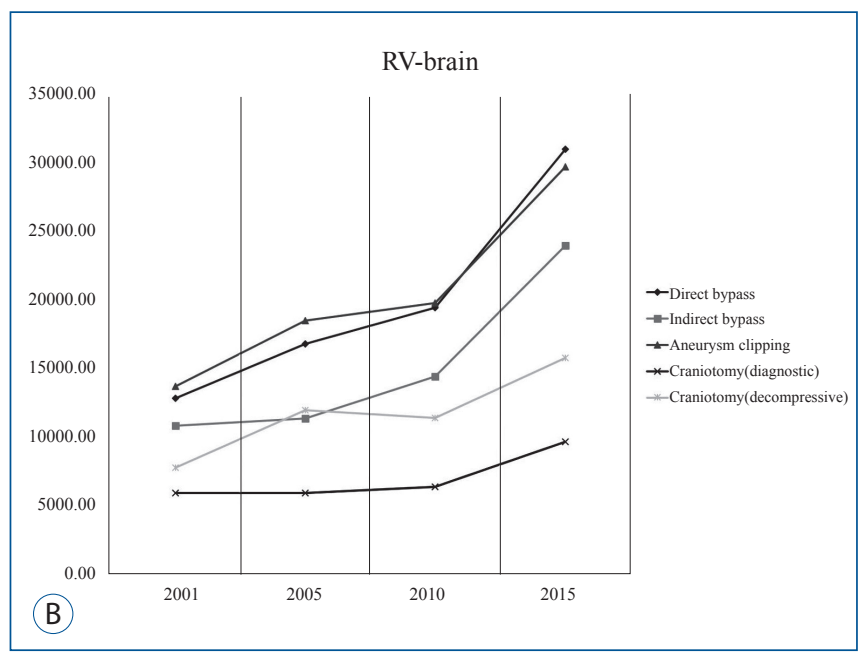

Fig. 3. Change of relative values of representative neurosurgical procedures during 15 years since the introduction of K-RBRVS. A : Change of relative values of tumor removal procedures. $B$ : Change of relative values of representative brain surgeries. $C$ : Change of relative values of representative spinal surgeries. K-RBRVS : Korean Resource Based Relative Value Scale, RV : relative value.

under K-RBRVS. And also compared the results with that of brain neurosurgical practices. Reimbursement to neurosurgeons, is categorized into three broad areas by the Korean Health Insurance Review \& Assessment Service (K-HIAS) which is not different from that of the United States' RBRVS $^{7}$. First, like all clinical specialties, diagnostic imaging studies as well as laboratory examinations provide revenue for neurosurgeons based on specific codes given by the KHIAS. Second, some minor outpatient department based simple procedures and some functional test or physical examinations have codes for reimbursement. Third, as we are surgeons, we earn revenue by performing operations performed in hospital based operating rooms.

While medical practices such as imaging studies or functional studies are the majority of practices in many non-surgical based specialties, the majority of reimbursement for 
Reimbursement of Neurospinal Precedures under K-RBRVS | Kwon WK, et al.

Table 2. RVs of representative surgical procedures in both Korea and USA, and the increase of RVs since 2003

\begin{tabular}{|c|c|c|c|c|c|c|}
\hline \multirow{2}{*}{ Neurosurgical field - spine } & \multicolumn{3}{|c|}{ Korea } & \multicolumn{3}{|c|}{ USA } \\
\hline & 2002 & 2015 & $\%$ & 2003 & 2015 & $\%$ \\
\hline Lumbar discectomy (including-laminotomy) & 6179 & 6500 & 105.2 & 55.25 & 66.12 & 119.7 \\
\hline 1 level posterior lumbar interbody fusion & 10570 & 11358 & 107.5 & 87.84 & 98.67 & 112.3 \\
\hline 1 level anterior cervical discectomy and fusion & 9273 & 14570 & 157.1 & 74.80 & 92.28 & 123.4 \\
\hline Cervical intramedullary tumor & 10260 & 25177 & 245.4 & 92.64 & 121.55 & 131.5 \\
\hline \multicolumn{7}{|l|}{ Neurosurgical field - brain } \\
\hline Supratentorial tumor & 11169 & 26389 & 236.3 & 128.75 & 157.37 & 122.2 \\
\hline Aneurysm clipping & 13716 & 29738 & 216.8 & 140.61 & 200.82 & 142.8 \\
\hline Direct intracranial vessel operation & 12816 & 31044 & 242.2 & 113.97 & 186.52 & 163.7 \\
\hline Decompressive craniectomy & 7767 & 15786 & 203.2 & 99.42 & 132.96 & 133.7 \\
\hline
\end{tabular}

$\mathrm{RV}$ : relative value

neurosurgeons come from surgical procedures. 143 out of $159(89.9 \%)$ coded practices in the neurosurgical field are surgical procedures, and the total amount of money reimbursed for these surgical procedures were 112 billion Korean Won, $76.8 \%$ of the total amount of reimbursement in $2013^{2}$. As surgeons, reimbursement for surgical procedures are surely of most interest.

In our study, we have found that the reimbursement for surgical procedures has been advantageous to brain surgeries than spinal surgeries. The gap of increase rates of RVs between two neurosurgical fields has been quite significant, as previously described (Table 1). The Korean neurosurgeons have a single large society, the Korean Neurosurgical Society (KNS) and both brain surgeons and spinal neurosurgeons are participating in this community. Although majority of the members are actually engaged in the spinal neurosurgical field, many policy decisions have been made mainly by brain neurosurgeons for a long period of time. The Chairman and President of this community has also been usually consisted of brain neurosurgeons much more often than spinal neurosurgeons. As brain neurosurgeons have played an active role in the society, policies related to the reimbursement for neurosurgical procedures might have also been mainly focused on brain surgeries, and spinal surgical procedures might have been relatively estranged from the main stream of policy interests. When comparing the actual increase of RVs in both neurosurgical fields between Korea and USA, we could rec- ognize how much the RVs of spinal procedures were estranged from proper increase. RVs of several frequently performed surgical procedures of both field in both nations are presented on Table 2. While the RV increase for such particular procedures were not so different between both fields in the USA, the increase rate was significantly higher for the brain procedures in Korea.

Another reason could be because of the unique circumstances of spinal surgical fields in Korea. Both neurosurgeons and orthopedic surgeons have their own society of spinal surgeons, and actually do not have an active academic exchange between societies. Nonetheless, the K-RBRVS have a single system of RVs for spinal surgical procedures, regardless of the spinal surgeon's specialty. Therefore, both societies should have closely collaborated and should have made united efforts in order to achieve adequate increase of RVs in the spinal surgical field, however they didn't for more than a decade. These complex circumstances probably resulted in a relatively low increase rate of RVs for spinal surgeries during the past 15 years.

As mentioned previously, RVs for the procedures we perform are determined on the basis of resource costs required to produce services: total work (time and intensity) of the physician, practice (overhead) costs and the opportunity costs regarding possible risks of professional negligence during procedures ${ }^{4,5)}$. Currently, the average proportion of physician's labor in total surgical practice RVs is $16 \%{ }^{3)}$. After the 
Table 3. Percentage of each costs planned for the second revision of K-RBRVS in representative neurosurgical procedural categories

\begin{tabular}{|c|c|c|c|c|}
\hline Neurosurgical field & Categories & Physician's labor (\%) & Practice costs (\%) & Opportunity costs (\%) \\
\hline \multirow[t]{7}{*}{ Brain } & Craniotomies & 26 & 61 & 12 \\
\hline & Cranial nerve surgeries & 18 & 74 & 9 \\
\hline & Endoscopic surgeries & 23 & 66 & 10 \\
\hline & Skull surgeries & 20 & 71 & 9 \\
\hline & Vascular surgeries & 20 & 70 & 10 \\
\hline & Stereotactic surgeries & 30 & 57 & 13 \\
\hline & Average & 22.8 & 66.5 & 10.5 \\
\hline \multirow[t]{5}{*}{ Spine } & Peripheral nerves & 20 & 76 & 4 \\
\hline & Spinal cord surgeries & 16 & 76 & 7 \\
\hline & Fusion/fixation surgeries & 17 & 77 & 6 \\
\hline & Discectomies & 19 & 72 & 8 \\
\hline & Average & 18.0 & 75.3 & 6.3 \\
\hline
\end{tabular}

K-RBRVS : Korean Resource Based Relative Value Scale

second major revision in K-RBRVS, the overall average for percentage of physician's labor in RVs are supposed to be $26 \%$ for surgical procedures ${ }^{2}$, which is much larger than before. But unfortunately, this trend of increase in the physician's labor doesn't seem that it will be reflected in the neurosurgical field. The percentage of each costs planned for the second revision of K-RBRVS in representative neurosurgical procedural categories of both brain and spinal surgeries are shown on Table 3. The average percentage of physicians' labor in RV will be $22.8 \%$ in brain surgeries while it will be only $18.0 \%$ in spinal surgeries ${ }^{2)}$. The opportunity cost representing possible risks of medical negligence is also lower in spinal surgical procedures than brain surgeries ${ }^{2)}(10.5 \%$ vs. 6.3\%). This result suggests that the K-HIAS estimates the spinal surgeons' labor much lower than that of brain surgeons, and also estimates that opportunity costs for risk of spinal procedure related issues are also much smaller than brain surgeries. The physicians' labor in brain neurosurgical procedures are lower than the average, and that of spinal neurosurgeries are even much more below the average. In the United States, the physician's labor takes $55 \%$ of the total $\mathrm{RV}^{1,6)}$ which is much higher than that of K-RBRVS which is $18 \%$ currently ${ }^{3)}$ (Fig. 4). The physician's work is defined as the physician time and intensity involved in providing a surgical procedure, and it includes mental effort, physical effort, technical skills and psychologic

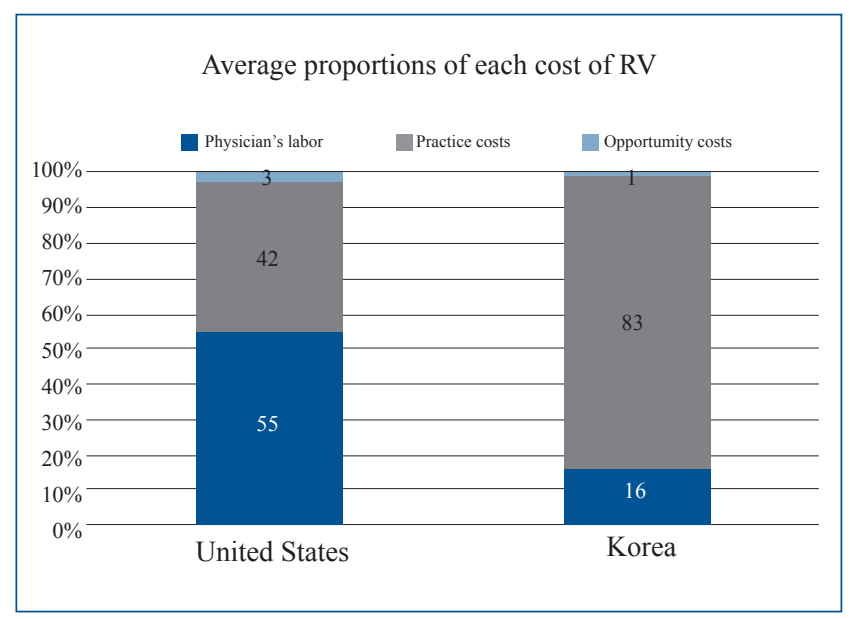

Fig. 4. Average proportion of physician's labor, practice costs and opportunity costs for RVs under Resource Based Relative Value Scale (RBRVS) of United States (US) and Korea. In the US, the physician's labor takes $55 \%$ of the total RV, which is much higher than that of Korean RBRVS which is $18 \%$ currently. RV : relative value.

stress involved in providing this surgical service ${ }^{6)}$. Although we do not have objective measures for this currently, however there surely is doubt in whether we are adequately reimbursed for these efforts or not. Especially more when we compare the proportion of physician's work with brain surgeons or that in the United States' RBRVS.

Finally, our study has found that the reimbursement for spinal surgical procedures have been relatively underestimated for more than a decade under the K-RBRVS. When we analyzed the RVs of spinal surgeries, they were significantly 
smaller than that of brain surgeries and the increase rate for 15 years was smaller as well. Another important point was that it was not only the overall RV of spinal surgeries that was underestimated, but also the proportion of surgeons' labor. Compared with that of United States' RBRVS, the underestimation of a spinal physician's labor was even more significant. Concerns that the RVs under RBRVS may not accurately reflect a surgeon's work, productivity or value is not surprising, and has already been an issue also in other surgical specialty societies ${ }^{8}$. The problem is, that not much attention has been paid for this problem for a long period of time in our surgical society and maybe this lack of interest for proper reimbursement might have led to this discrimination of RVs. As the second major revision for K-RBRVS has already been done and is about to be under force within this year, it might be quite late to start any effort in order to fix this problem, and gain impartial reimbursement within a short period of time. However, it is surely better late than never, and effort trying to reduce the gap of increase in RVs between spinal surgeries and brain surgeries should be done as soon as possible. Having regular policy meetings with the K-HIAS and effort to let them understand the current discrimination, and let them know our demands for proper reimbursement should be done. We should also show our effort in developing more objective measures of surgeon work to make the proportion of physician's work more adequate. And additionally, effort to collaborate closely with the orthopedic spinal surgeons to achieve this matters of mutual concern will be necessary as well.

\section{CONCLUSION}

During the past 15 years, RVs for spinal neurosurgical pro- cedures have increased in a rate of $62.8 \%$, however this is hardly an actual increase when compared to the cumulative increase of consumer prices during this time period or the increase rate of $92.3 \%$ for brain surgeries. More effort of the society of spinal surgeons seems to be needed to get adequate reimbursement, as there have been some discrimination compared to brain surgeons in the increase of RVs. And considering the relative underestimation of spinal neurosurgeons' labor, more objective measures of neurospinal surgeons' work and productivity should be developed for impartial reimbursement.

\section{References}

1. 2015 National Physician Fee Schedule Relative Value File. American Medical Association. Chicago (IL), USA : Centers for Medicare \& Medicaid Services, 2015

2. Current strategies of the second revision of Korean ResourceBased Relative Value Scale : Presentation for the Korean Spinal Neurosurgery Society. Seoul, Korea : Korean Health Insurance Review \& Assessment Service, 2015

3. National Health Insurance Physician Fee Schedule Relative Value. Korean Hospital Association. Seoul, Korea : Korean Hospital Association, 2015

4. Hsiao WC, Braun P, Dunn DL, Becker ER, Yntema D, Verrilli DK, et al. : An overview of the development and refinement of the resource-based relative value scale. The foundation for reform of U.S. physician payment. Med Care 30(11 Suppl) : NS1-12, 1992

5. Kwon $S$ : Payment system reform for health care providers in Korea. Health Policy Plan $18:$ 84-92, 2003

6. Laugesen MJ : The resource-based relative value scale and physician reimbursement policy. Chest 146 : 1413-1419, 2014

7. Monk BJ, Burger RA : Reimbursement for surgical procedures in gynecologic oncology. Curr Opin Oncol 13 : 390-393, 2001

8. Shah DR, Bold RJ, Yang AD, Khatri VP, Martinez SR, Canter RJ : Relative value units poorly correlate with measures of surgical effort and complexity. J Surg Res 190 : 465-470, 2014 